\section{Air Pollution and Liver Enzymes}

To the Editor:

rowing evidence indicates that elevated levels of the liver enzymes $\gamma$-glutamyltransferase (GGT), aspartate transaminase (AST), and alanine transaminase (ALT) are independently associated with increased risk of cardiovascular disease (CVD). ${ }^{1-4}$ GGT may also increase due to environmental pollution. ${ }^{1}$ Ambient particulate matter has been shown to induce oxidative stress and being linked to $\mathrm{CVD}^{5}$ and might potentially affect liver enzymes' levels. Therefore, we assessed the association between chronic ambient air pollution and serum liver enzymes, as a possible component in the mechanisms linking air pollution to CVD.

We analyzed data collected in two KORA (Cooperative Health Research in the Region of Augsburg) surveys, conducted in Augsburg and two adjacent counties in southern Germany between 2004 and 2008. Blood was drawn from 5,892 adults aged 31 to 85 years, and the serum liver enzymes GGT, AST, and ALT were analyzed. Air pollution exposure was estimated within the ESCAPE study (European Study of Cohorts for Air Pollution Effects, http://www.escapeproject.eu/) between 2008 and 2009 by a combination of measurements and modeling. We estimated the annual

Supported by funding from the European Community's Seventh Framework Program (FP7/20072011) under grant agreement number: 211250.

The Cooperative Research in the Region of Augsburg (KORA) research platform was initiated and financed by the Helmholtz Zentrum München - German Research Centre for Environmental Health, which is funded by the German Federal Ministry of Education and Research and by the state of Bavaria.

SDC Supplemental digital content is available through direct URL citations in the HTML and PDF versions of this article (www.epidem. com). This content is not peer-reviewed or copyedited; it is the sole responsibility of the author.

Copyright (C) 2013 by Lippincott Williams \& Wilkins

ISSN: 1044-3983/13/2406-0934

DOI: 10.1097/EDE.0b013e3182a77600

TABLE. Percent Change (95\% Cl) of Mean Live Enzymes per 5\%-95\% Range Increase in Air Pollutants in the Augsburg Area, Germany (2004-2009)

\begin{tabular}{lccrr}
\hline Pollutant & $\mathbf{5 \% - 9 5 \%}$ & $\begin{array}{c}\text { GGT of Change } \\
\text { (95\% CI) }\end{array}$ & $\begin{array}{c}\text { AST of Change } \\
\text { (95\% CI) }\end{array}$ & $\begin{array}{c}\text { ALT of Change } \\
\text { (95\% CI) }\end{array}$ \\
\hline $\mathrm{PM}_{2.5}\left(\mu \mathrm{g} / \mathrm{m}^{3}\right)$ & 2.8 & $5.1(0.1$ to 10.4$)$ & $0.7(-1.6$ to 3.0$)$ & $-1.0 .(-4.4$ to 2.6$)$ \\
$\mathrm{PM}_{10}\left(\mu \mathrm{g} / \mathrm{m}^{3}\right)$ & 7.7 & $3.8(-1.3$ to 9.1$)$ & $0.3(-2.0$ to 2.7$)$ & $0.6(-3.0$ to 4.2$)$ \\
$\mathrm{PM}_{\text {coarse }}\left(\mu \mathrm{g} / \mathrm{m}^{3}\right)$ & 3.5 & $3.5(-1.7$ to 8.9$)$ & $-0.4(-2.7$ to 2.0$)$ & $0.4(-3.2$ to 4.1$)$ \\
$\mathrm{PM}_{2.5}$ absorbance $\left(10^{-5} \mathrm{~m}^{-1}\right)$ & 0.5 & $1.9(-3.1$ to 7.1$)$ & $-0.4(-2.6$ to 2.0$)$ & $-1.0(-4.5$ to 2.6$)$ \\
$\mathrm{NO}_{\mathrm{x}}\left(\mu \mathrm{g} / \mathrm{m}^{3}\right)$ & 22.3 & $2.5(-2.4$ to 7.6$)$ & $-0.2(-2.4$ to 2.1$)$ & $1.2(-2.3$ to 4.7$)$ \\
$\mathrm{NO}_{2}\left(\mu \mathrm{g} / \mathrm{m}^{3}\right)$ & 11.8 & $3.0(-2.0$ to 8.1$)$ & $0.5(-1.8$ to 2.8$)$ & $2.5(-1.0$ to 6.1$)$ \\
\hline
\end{tabular}

$\mathrm{CI}$ indicates confidence interval.

average concentrations of particles below $2.5 \mu \mathrm{m}\left(\mathrm{PM}_{2.5}\right)$, below $10 \mu \mathrm{m}\left(\mathrm{PM}_{10}\right)$, coarse particles $\left(\mathrm{PM}_{\text {coarse }}\right)$, absorbance of $\mathrm{PM}_{2.5}$, nitrogen oxides $\left(\mathrm{NO}_{\mathrm{x}}\right)$, and nitrogen dioxide $\left(\mathrm{NO}_{2}\right)$ at the residential address of each participant. We assessed the associations by multivariable linear models with log-transformed outcome variables. All models were adjusted for socioeconomic, lifestyle, and clinical covariates. For a detailed description of the outcome and exposure variables as well the covariates see the eAppendix (http://links.lww.com/EDE/A717).

Percent changes of liver enzymes means associated with an increase in air pollutants from $5 \%$ of the distribution to $95 \%$ are shown in the Table. For GGT, elevated levels of pollutants were associated with increased mean serum level, most strongly for $\mathrm{PM}_{2.5}$. An increase of the annual average concentration of $\mathrm{PM}_{2.5}$ at residences of $2.77 \mu \mathrm{g} / \mathrm{m}^{3}$ (5\%-95\% range) increased mean serum concentration of GGT among the study participants by $5.1 \%$ $(95 \%$ confidence interval $=0.1 \%$ to $10.4 \%)$. The association was stronger for participants with CVD (12.0\% [4.4\% to $20.2 \%]$ ), whereas those without CVD showed no association. For AST and ALT, we observed no consistent patterns.

One biological insight into the association of serum GGT and CVD induction is a possible role of GGT in oxidative stress. ${ }^{1}$ GGT is present in atherosclerotic plaques and may catalyze oxidation of low-density lipoproteins, contributing to plaque evolution and rupture. ${ }^{1}$ Also, GGT acts as a protein catalyst in the catabolism of glutathione, the major thiol antioxidant in the body. ${ }^{1}$ The role of GGT in oxidative stress and in the progression of atherosclerosis has been supported by the association with carotid intima-media thickness. ${ }^{2,4,6}$ Moreover, GGT is more strongly associated with cardiovascular outcomes than ALT, ${ }^{1,7}$ which is considered to be a marker of liver injury but not of oxidative stress. ${ }^{1}$

Thus, our findings concerning GGT may strengthen the hypothesis that particulate air pollution affects the cardiovascular system through mechanisms related to systemic oxidative stress. As correlations between $\mathrm{PM}_{2.5}$ and other pollutants were weak, this suggests that the pathway of $\mathrm{PM}_{2.5}$ might differ from the pathways of other pollutants. For example, $\mathrm{PM}_{2.5}$ can penetrate deeper into the pulmonary tree compared with $\mathrm{PM}_{10}$ or $\mathrm{PM}_{\text {coarse }}$ because of its smaller aerodynamic diameter. Additionally, our finding regarding strong effect modification by CVD might indicate that people with CVD are more susceptible to air pollution. However, as our reported association has not been assessed previously, it has to be replicated in other studies.

Iana Markevych
Ludwig-Maximilians-Universität
(LMU) Munich
and Epidemiology
Munich, Germany

iana.markevych@helmholtz-muenchen.de 


\section{Kathrin Wolf Regina Hampel Susanne Breitner Alexandra Schneider Stephanie von Klot Josef Cyrys \\ Helmholtz Zentrum München German Research Center for Environmental Health Institute of Epidemiology II Neuherberg, Germany \\ Joachim Heinrich Angela Döring \\ Helmholtz Zentrum München German Research Center for Environmental Health Institute of Epidemiology I Neuherberg, Germany}

Rob Beelen Institute for Risk Assessment Sciences (IRAS) Utrecht University Utrecht, The Netherlands

Wolfgang Koenig University of Ulm Medical Center Department of Internal Medicine II-Cardiology Ulm, Germany

Annette Peters

Helmholtz Zentrum München German Research Center for Environmental Health Institute of Epidemiology II Neuherberg, Germany

\section{REFERENCES}

1. Targher G. Elevated serum gamma-glutamyltransferase activity is associated with increased risk of mortality, incident type 2 diabetes, cardiovascular events, chronic kidney disease and cancer - a narrative review. Clin Chem Lab Med. 2010;48:147-157.

2. Bellentani S, Bedogni G, Tiribelli C. Liver and heart: a new link? J Hepatol. 2008;49: 300-302.

3. Wang CC, Lin SK, Tseng YF, et al. Elevation of serum aminotransferase activity increases risk of carotid atherosclerosis in patients with nonalcoholic fatty liver disease. $J$ Gastroenterol Hepatol. 2009;24:1411-1416.

4. Abdou AS, Magour GM, Mahmoud MM. Evaluation of some markers of subclinical atherosclerosis in Egyptian young adult males with abdominal obesity. Br J Biomed Sci. 2009;66:143-147.

5. Brook RD, Rajagopalan S, Pope CA 3rd, et al.; American Heart Association Council on Epidemiology and Prevention, Council on the Kidney in Cardiovascular Disease, and Council on Nutrition, Physical Activity and Metabolism. Particulate matter air pollu- tion and cardiovascular disease: An update to the scientific statement from the American Heart Association. Circulation. 2010;121: 2331-2378.

6. Koskinen J, Magnussen CG, Kähönen M, et al. Association of liver enzymes with metabolic syndrome and carotid atherosclerosis in young adults. The Cardiovascular Risk in Young Finns Study. Ann Med. 2012;44: 187-195.

7. Ghouri N, Preiss D, Sattar N. Liver enzymes, nonalcoholic fatty liver disease, and incident cardiovascular disease: a narrative review and clinical perspective of prospective data. Hepatology. 2010;52:1156-1161.

\section{Relative Risks from Case-Population Data}

\section{To the Editor:}

$\mathrm{T}$ he usual designs to assess the relationship between exposure and outcome are cohort studies, resulting in relative risks (RRs), and casecontrol studies, resulting in odds ratios (ORs). Traditionally case-control studies are preferred when events are rare, and cohort studies when exposures are uncommon. When both events and exposures are rare, there is a particular challenge. In such settings, the casepopulation approach might be of help.

Case-population studies compare exposure in cases and in the general population. ${ }^{1,2}$ This design requires exhaustive or representative collation of cases of interest in a given territory and a measure of exposure to the exposure of interest in the territory's population. Denominator is person-time, ${ }^{2}$ or number of exposed subjects. ${ }^{3}$ The metric is the case-population ratio, the ratio of exposure in cases and in the general population.

The SALT study from which some of the data used was done at the request of the European Medicines Agency and funded by an unrestricted grant from Helsinn Healthcare. This work was unfunded, and the authors report no conflict of interest.

SDC Supplemental digital content is available through direct URL citations in the HTML and PDF versions of this article (www.epidem.com). This content is not peer-reviewed or copy-edited; it is the sole responsibility of the author.

Copyright (C) 2013 by Lippincott Williams \& Wilkins

ISSN: $1044-3983 / 13 / 2406-0935$

DOI: 10.1097/EDE.0b013e3182a77882
The case-population ratio is perpendicular to the RR: rather than the ratio of case rates among exposed and unexposed, it is the ratio of exposure rates among cases and population. In the usual two-by-two table (Table), where $a$ is the number of exposed cases, $b$ the number of exposed noncases, $c$ the number of unexposed cases, and $d$ the number of unexposed noncases, RR is $(a / a+b) /$ $(c / c+d)$, OR is $a / c / b / d$ (or $a d / b c$ ), and case-population ratio is $(a / a+c) /((a+b) /$ $(a+b+c+d))$. The analysis population may come from a representative population sample with a known sampling rate or from representative samples with unknown sampling rates (eg, the UK Clinical Practice Research Datalink). In that case, case-population ratio could be expressed as $(a /(a+c)) /((e / e+f))$, where $\mathrm{e}$ and $\mathrm{f}$ are the exposed and unexposed in the sample, which may or not include the cases.

If cases are rare, case-population ratio can be simplified to $a d / b c /$ $((1-$ Pexp $) /(1-C \exp ))$, where Pexp is the population exposure to the drug of interest $(b / b+d)$, and Cexp is the case exposure to the drug of interest $(a / a+c)$. The smaller the population and case exposures, the better case-population ratio (CPR) approximates the OR. The OR estimates the RR when the event is rare, so the lower the exposure in cases and in the general population, and the rarer the event, the better the CPR approximates the real RR of the association of exposure and event.

We built a table of CPR for various RR and population exposures (eTable, http://links.lww.com/EDE/A718). When population exposure is below $1 \%$, the difference between case-population ratio and actual OR or RR is less than $1 \%$. Above 1\%, CPR underestimates RR above 1 and overestimates RR below 1 .

We tested this in a case-population study of liver transplantation in Europe for which drug utilization was the exposure of interest. ${ }^{3-5}$ In this study with exhaustive case identification, and full description of the country's drug utilization over the same period and in the same population of patients, we computed the actual 